Research Paper

\title{
Long non-coding RNA TCL6 enhances preferential toxicity of paclitaxel to renal cell carcinoma cells
}

\author{
Zhizhao Chen ${ }^{1,2}$, Quan Zhuang ${ }^{1}$, Ke Cheng', Yingzi Ming1, Yujun Zhao1, Qifa Ye ${ }^{1,2}$, Sheng Zhang ${ }^{\circledR}$ \\ 1. The Third Xiangya Hospital of Central South University, Changsha, China; \\ 2. Wuhan University, Zhongnan Hospital of Wuhan University, Institute of Hepatobiliary Diseases of Wuhan University, Transplant Center of Wuhan \\ University, Hubei Key Laboratory of Medical Technology on Transplantation, Wuhan Hubei, China. \\ $\bowtie$ Corresponding author: Sheng Zhang, The Third Xiangya Hospital of Central South University, Changsha, China. E-mail: zhangsxy3@aliyun.com
}

( ) The author(s). This is an open access article distributed under the terms of the Creative Commons Attribution License (https://creativecommons.org/licenses/by/4.0/). See http://ivyspring.com/terms for full terms and conditions.

Received: 2018.12.24; Accepted: 2019.10.26; Published: 2020.01.01

\begin{abstract}
Background: Recent findings have shown long non-coding RNAs (IncRNAs) are dysregulated in a variety of cancer cells. In this report, we investigate the effect of T-cell leukemia lymphoma 6 (TCL6) on paclitaxel (PTX)-induced apoptosis in Renal cell carcinoma (RCC) cells.

Methods: Expression levels of TCL6 in RCC tissues were analyzed via The Cancer Genome Atlas (TCGA) and Gene Expression Omnibus (GEO) datasets. Fluorescence in situ hybridization (FISH) was performed to detect the expression of TCL6 in RCC tissues and cells. Two pairs of cell lines were used: TCL6-silenced 786-O cell line and scrambled 786-O cell line, TCL6-overexpressed Caki-1 cell line and Caki-1 scrambled cell line. Cell viability was detected using the MTT assay. Apoptosis was examined by flow cemetery. Dual reporter gene assay was performed to confirm the direct downstream target miRNA of TCL6.

Results: Based on RNA sequencing expression data of RCC tissues from TCGA and GEO datasets, the expression deficiency of TCL6 was observed in RCC tissues. Low level of TCL6 was associated with worse overall and disease-free survival of RCC patients. The FISH showed similar results with low expression of TCL6 in RCC tissues and cells. After PTX treatment, a time-dependent decrease in cell viability was observed in TCL6-overexpressed RCC cells and an increase in cell viability was observed in TCL6-silenced cells compared to control cells. Apoptosis induced by PTX was significantly increased in TCL6-overexpressed cells. Inhibition of TCL6 showed a significant decrease in apoptosis. Furthermore, luciferase reporter assay revealed that TCL6 is a direct target gene of miR-221.

Conclusions: TCL6 effectively sensitizes RCC to PTX mainly through downregulation of miR-221. Our results suggest that PTX combined with TCL6 might be a potentially more effective chemotherapeutic approach for renal cancer.
\end{abstract}

Key words: Renal cell carcinoma, Apoptosis, Paclitaxel, Chemotherapy.

\section{Introduction}

RCC is an epithelial tumor derived from the proximal tubules of the nephrons and it is the most common primary tumor of the renal parenchyma, accounting for approximately $90 \%$ of kidney neoplasms and $3 \%$ of all malignancies [1,2]. The most common histological RCC type is the clear cell RCC, which is estimated as $80 \%$ of all patients [3]. The currently available therapeutic options including chemotherapy, radiotherapy, targeted therapy, and immunotherapy are ineffective and the clinical management of advanced RCC is still challenging [4, 5]. A primary cause of the resistance and subsequent treatment failure is RCC intra-tumoral heterogeneity [6-8]. Targeting common elements between different molecular/genetic subclasses of RCC may represent a potential therapeutic strategy.

LncRNAs are a highly heterogeneous group of untranslated RNA molecules with over 200 nucleotides but lack open reading frames [9]. LncRNAs are frequently aberrantly expressed in 
multiple cancers having regulatory roles in fundamental biological processes, which function as oncogenes or suppressor genes in tumor initiation and progression. Recent studies of RCC have reported lncRNA expression profiles by gene microarray analysis and identified the roles of specific lncRNAs such as HOTAIR [10], MALAT1 [11], GAS5 [12] and LINC00961 [13]. However, the detail function of lncRNAs in RCC remains largely unknown. TCL6, also named TNG1 or TNG2, located on 14q32.13. A recent study demonstrated that decreased expression of TCL6 was associated with poor prognosis in patients with RCC [14].

PTX is an effective mitotic inhibitor and apoptosis inducer. It has been widely used in chemotherapy for multiple cancers [15]. How to enhance the sensitivity of renal cancer cells to PTX is still a problem that needs to be solved.

TCGA, a public available platform with more than 30 types of cancer from 11,000 patients at least and their clinicopathological information, has been widely applied by large numbers of researches to explore the genetic basis of tumor according to the high-throughput sequencing [16]. In the current study, we found the expression deficiency of TCL6 was observed in the RCC tissues through analysis of TCGA and GEO databases. Low level of TCL6 was associated with worse overall and disease-free survival of RCC patients. These findings hint that TCL6 plays an important role in the progression of RCC. We aimed to elucidate the potential mechanisms of TCL6 modulating survival of cancer cells after PTX treatment.

\section{Materials and methods}

\section{TCGA dataset of RCC clinical samples}

The online data of TCL6 expression level, clinical and prognosis characteristics were analyzed by Gene Expression Profiling Interactive Analysis (GEPIA) (http://gepia.cancer-pku.cn/detail.php \#\#\#), The Atlas of Noncoding RNAs in Cancer (TANRIC) platforms (https://ibl.mdanderson. org/tanric/_design/basic /query.html) and GEO datasets. GEPIA and TANRIC are web servers for analyzing the RNA sequencing expression data from the TCGA database. Search strategies were as previous report $[17,18]$.

10 cases of human RCC tissues and adjacent normal renal tissues were formalin-fixed and paraffin-embedded. All specimens were collected from the Third Xiangya Hospital of Central South University.

\section{Cell culture}

Human renal proximal tubule epithelial cell line
(HK-2) and RCC cell lines (OS-RC-2, KC, ACHN, 786-O, and Caki-1) were purchased from American Type Culture Collection (ATCC). HK-2 cells were cultured in DMEM/F12 containing 10\% FBS. 786-O cells were cultured in 1640 medium supplemented with $10 \% \mathrm{FBS}$ at $37^{\circ} \mathrm{C}$ with $5 \% \mathrm{CO}_{2}$ in a humidified atmosphere. OS-RC-2, KC, ACHN, and Caki-1 cells were cultured in DMEM/10\% FBS medium.

\section{Fluorescence in situ hybridization (FISH)}

All specimens and cell lines were subjected to FISH for TCL6 expression using the FISH kit (C10910; Ribobio, Guangzhou, China). The red FISH Probe of TCL6 (Lnc1CM001， 5'-CTATCCATTCAGCATCAG AGA-3'), U6 (Lnc110101, internal reference) and 18S (Lnc110201) were purchased from Ribobio (Guangzhou, China). Briefly, pre-hybridization buffer was added to unstained RCC tissue sections for 30 min. And then TCL6 FISH Probe Mix was added to each section and left overnight. Nuclear was stained by blue DAPI incubation at room temperature for 10 min. Cells $\left(5 \times 10^{4} /\right.$ well $)$ were seeded in 24 -well plate and then fixed by $4 \%$ paraformaldehyde. $200 \mu \mathrm{L}$ of pre-hybridization solution was added in each well for $30 \mathrm{~min}$. $200 \mu \mathrm{L}$ of hybridization solution was then added, and incubation at room temperature overnight. Fluorescence microscopy was used to capture images.

\section{TCL6 siRNA and overexpression plasmid transfection}

TCL6 was inserted the pcDNA3.1 plasmid (Invitrogen, Carlsbad, CA, USA) by Yingrun Company (Changsha, China). Knockdown of TCL6 was performed using short interfering (si) RNAs provided by Guangzhou Ribobio Co., Ltd. (Guangzhou, China). The siRNA sequences were as follows: si1-TCL6: 5'-CCTTAACCTTGGCACA ACA-3', si2-TCL6 forward: 5'-ACTCACAG AGGA GCAGAAT-3', and si3-TCL6 forward: 5'-GCAAT CAGAAACAGAAAGT-3'. scrambled control siRNAs were synthesized by Ribobio (Guangzhou, China). Cells were plated in 6-well plates. Transfection was performed when cells reached $80 \%$ confluence using $10 \mu \mathrm{l}$ Lipofectamine ${ }^{\circledR} 2000$ (Thermo Fisher Scientific). To validate the efficiency of transfection, lncRNA expression was examined by fluorescence microscope and qRT-PCR.

\section{Quantitative real-time RT-PCR.}

Total RNA from cells and tissues were extracted with TRIzol reagent (Invitrogen; Thermo Fisher Scientific, Inc) according to the manufacturer's protocol. SuperScript II Reverse Transcriptase kit (Invitrogen Life Technologies) was used to 
generate cDNA. Quantitative real-time PCR analyses were performed according to previous study [19]. Samples were compared using the relative CT method, where the relative expression of TCL6 was normalized to that of GAPDH. U6 served as internal control for the quantification of miR-221. The primers were synthesized by Ribobio (Guangzhou, China) and the sequences as follows: TCL6 forward, 5'-CTAT CCATTCAGCATCAGAGA-3' and reverse, 5'-CAC ATACTCACGCATCCTT-3' (Ribobio, Guangzhou, China). GAPDH forward, 5'-ATGACATCAAGA AGGTGG TG-3' and reverse, 5'-CATACCAGGA AATGAGCTTG-3' (Ribobio, Guangzhou, China).

\section{Cell proliferation assay.}

The MTT assay was used to measure cell proliferation after PXT (Beyotime, Shanghai, China) treatment. Cells $\left(5 \times 10^{3}\right)$ were seeded in 96-well plates and cultured for $24,48,72$ and $96 \mathrm{~h}$, and then the supernatant was removed and MTT ( $5 \mathrm{mg} / \mathrm{ml}, 20 \mu \mathrm{l}$, Beyotime, Shanghai, China) was added to each well at $37^{\circ} \mathrm{C}$. After $4 \mathrm{~h}, 100 \mu \mathrm{l}$ DMSO was added to each well. The OD value was determined at $490 \mathrm{~nm}$ by a microplate reader and used to construct a growth curve to assess cell proliferation.

\section{Apoptosis analysis}

Apoptosis was evaluated using Annexin V-FITC kit (Beyotime, Shanghai, China), Cells $\left(2 \times 10^{6}\right)$ were seeded in T25 cell culture flask and incubated for $48 \mathrm{~h}$. Cells were suspended in $195 \mu \mathrm{L}$ Annexin V-FITC binding buffer. $5 \mu \mathrm{L}$ of Annexin V-FITC and $10 \mu \mathrm{L}$ of propidium iodide (PI) were added and then incubated for $20 \mathrm{~min}$ at $25^{\circ} \mathrm{C}$ in the darkroom. The percentage of apoptotic cells was evaluated using a flow cytometer.

\section{Dual-luciferase reporter assays}

Luciferase reporter assays were performed using the pmirGLO-TCL6-Luc vector. HEK293 cells (ATCC, Manassas, VA, USA) were co-transfected with pmirGLO-TCL6-Luc vector $\left(\begin{array}{lll}1 & \mu \mathrm{g}\end{array}\right)$ and miR-221-mimics or NC-mimics (40 pmol). Cells were harvested $48 \mathrm{~h}$ after transfection and analyzed with the dual-luciferase reporter gene assay kit E1910 (Promega). The firefly luciferase values were normalized to Renilla luciferase values as an internal control.

\section{Statistical analysis.}

All experiments were repeated at least three times. Statistical analyses were carried out using SPSS 19.0 software (SPSS, Chicago, IL, USA). The results were assessed by Student's t-test or one-way analysis of variance. All statistical tests were two-sided, and a P-value of $<0.05$ was considered to indicate a statistically significant difference.

\section{Results}

\section{The expression deficiency of TCL6 was observed in RCC tissues}

To elucidate expression levels of TCL6 in RCC tumorigenesis, the GEPIA platform was performed to comprehensively analyze the TCL6 in 523 cases of RCC tissues and 100 cases of normal renal tissues. The results revealed that TCL6 was significantly decreased in cancer versus normal tissues (Fig.1A). These data indicate that TCL6 may regulate tumorigenesis and development of RCC. We next analyzed the expression levels of TCL6 using TANRIC platform. The results of the analysis showed that the TCL6 was significantly down-regulated in 448 cases of RCC tissues as compared to 67 cases of normal renal tissues (Fig.1B). We analyzed the TCL6 expression in 72 cases of fresh RCC and adjacent normal tissues from GEO dataset \#GSE53757. The data indicate that the TCL6 expression was lower in RCC tissues than adjacent normal tissues (Fig.1C). And TCL6 expression is progressively reduced in advancing clinical stages of renal cancer (Fig.1D). Low level of TCL6 was associated with worse overall (Fig.1E, G) and disease free survival (Fig.1F) of RCC patients.

\section{TCL6 inhibits proliferation after PTX treatment}

We further examined the expression level of TCL6 in human RCC tissues and cells using FISH. The FISH showed similar results with low expression of TCL6 in RCC tissues compared to the adjacent normal renal tissues (Fig.2A). LncRNA TCL6 was mainly located in the cytoplasm. We further detected the expression of TCL6 in human renal proximal tubule epithelial cell line (HK-2) and RCC cell lines (OS-RC-2, KC, ACHN, 786-O, and Caki-1). TCL6 was down-regulated in all RCC cell lines compared to the HK-2 cells as evidenced by FISH (Fig.2B). 786-O and Caki-1 cells were used for further experiments based on their showed lower expression of TCL6 compared to OS-RC-2, KC, ACHN cells. As shown in Fig.2C, the expression of TCL6 was downregulated in 786-O and Caki-1 cells compared to HK-2 cells as evidenced by qRT-PCR.

Our findings and a previous study [14] suggested that TCL6 plays an important role in the progression of RCC. But the impact of TCL6 on RCC cell PTX chemotherapy is unclear. To investigate this issue, we modify the expression level of TCL6 and analyzed its impacts on the cell viability of 786-O and Caki-1 cells with PTX. 
A

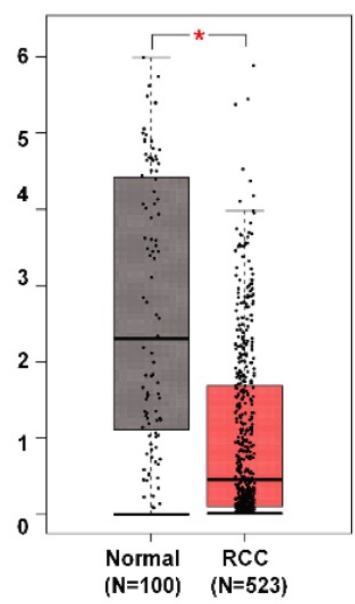

D

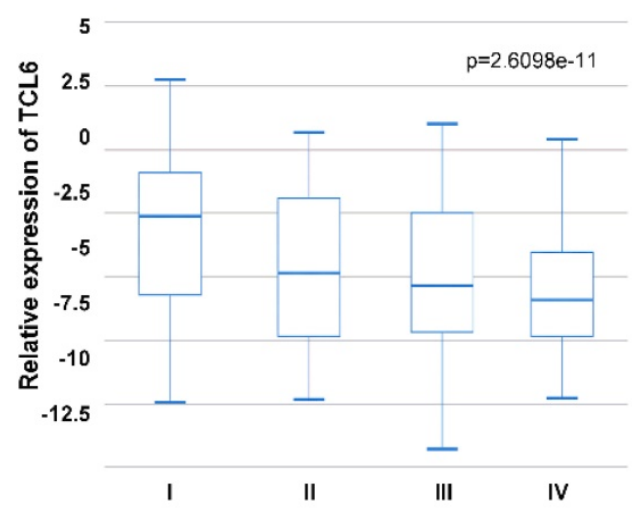

G
B

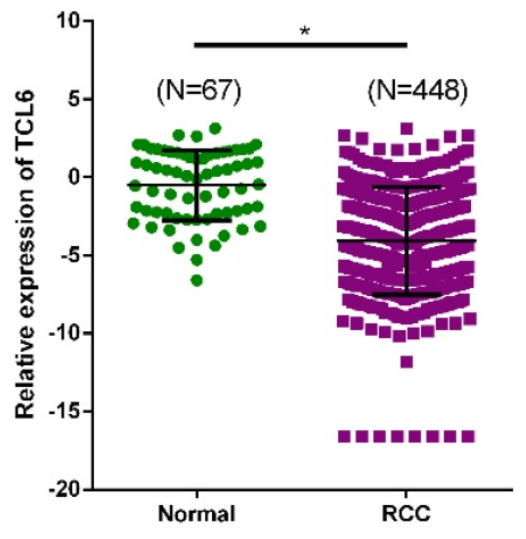

E

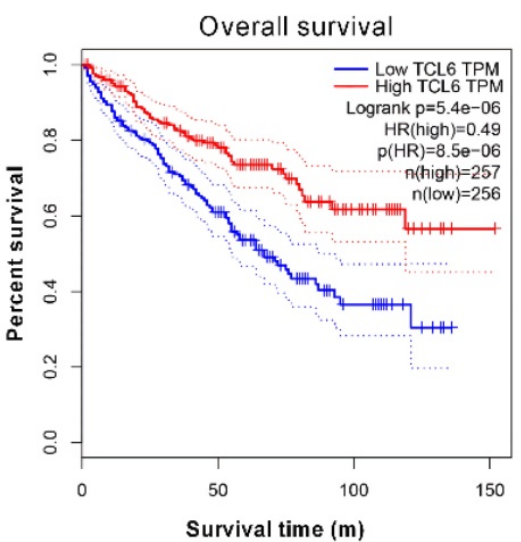

C

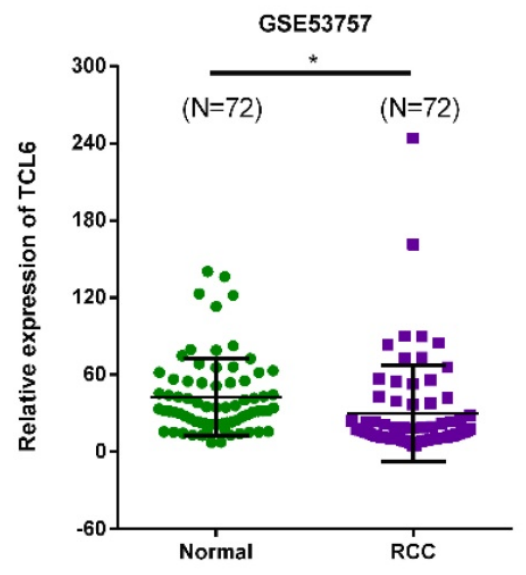

F

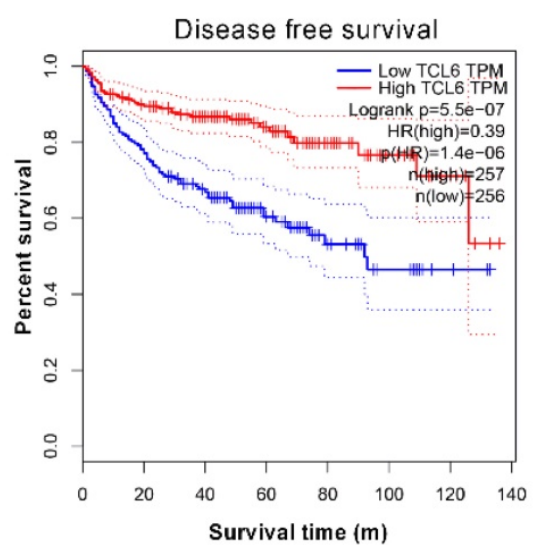

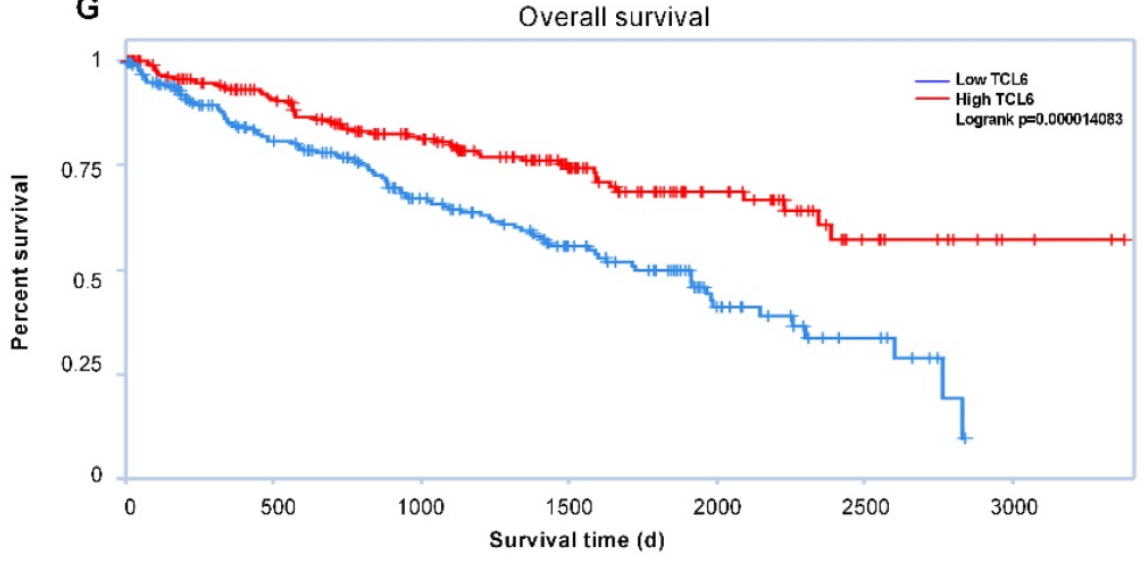

Figure 1. The downregulation of TCL6 expression was associated with poor prognosis of patients. A. The expression of TCL6 was decreased in RCC tissues ( 523 cases) compared to the normal tissues (100 cases), which of data was collected from the TCGA database and analyzed by GEPIA platform. B. The TCL6 expression data of RCC $(n=448)$ and normal $(n=67)$ tissues were further analyzed by online TANRIC platform. C. We further analyzed the expression levels of TCL6 in seventy-two RCC and match normal tissues obtained from the GEO database (GSE53757dataset). D. The expression of TCL6 gradually decreased among the different clinical stages of renal cancer (by one-way analysis of variance). Data are shown as means \pm SD. Kaplan-Meier curves showed the patient overall survival (E) and disease-free survival (F) of the high TCL6 expression and low TCL6 expression. These graphs were conducted by TANRIC platform. Dotted line: the 95\% confidence interval $(\mathrm{Cl})$. G. Low level of TCL6 expression was associated with poor overall survival of patients with renal cancer. $* P<0.05$.

Three siRNAs target TCL6 were transfected into 786-O cells, and the third one revealed the best knockdown effect as evidenced by qRT-PCR (Fig.2B). The results of qRT-PCR show that TCL6 expression in
Caki-1 cells was notably increased after transfection with TCL6 overexpressing plasmid (Fig.2C). The cell viability was analyzed by MTT assay after being treated with various concentrations of PTX. As shown 
in Fig.3A, the cytotoxicity of PTX in TCL6 deficient 786-O cells was reduced compared to control 786-O cells. Suppression of cell growth by PTX on TCL6 overexpressing Caki-1 cells was more significant than that of control Caki-1 cells (Fig.3B), indicating more severe PTX-induced cytotoxicity to TCL6-enriched cells.

\section{TCL6 induces apoptosis after PTX treatment}

PTX is an effective apoptosis inducer. To further investigate whether TCL6 exerts synergistic

A
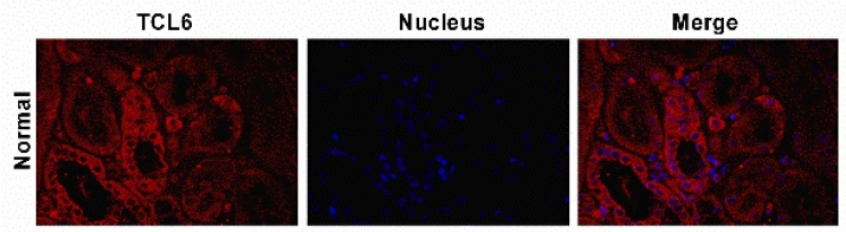

B
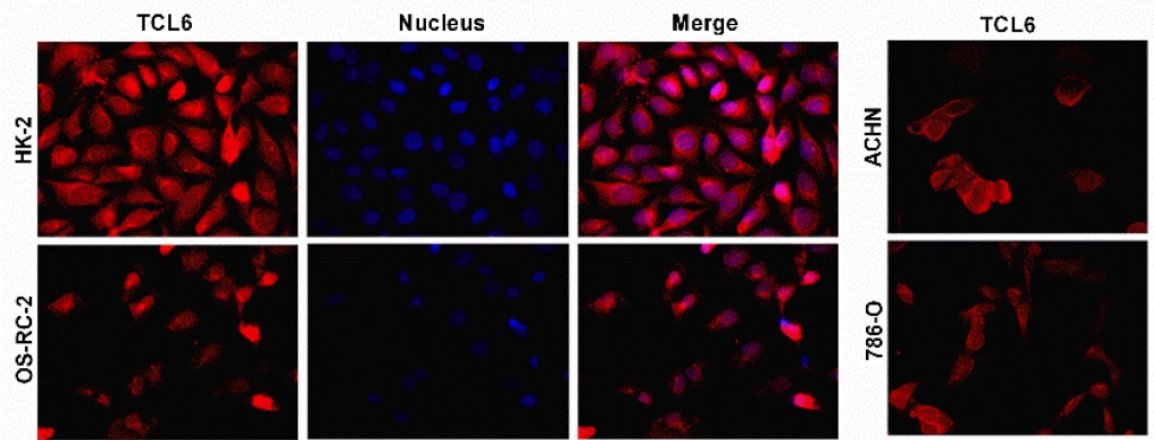

C

D

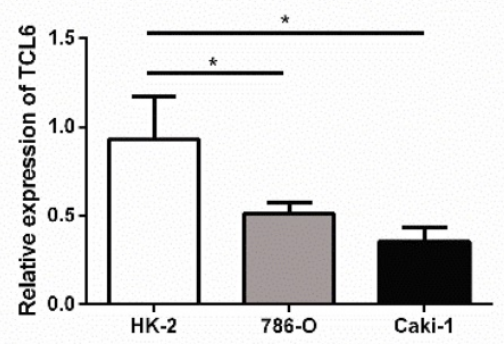

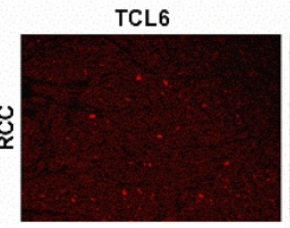
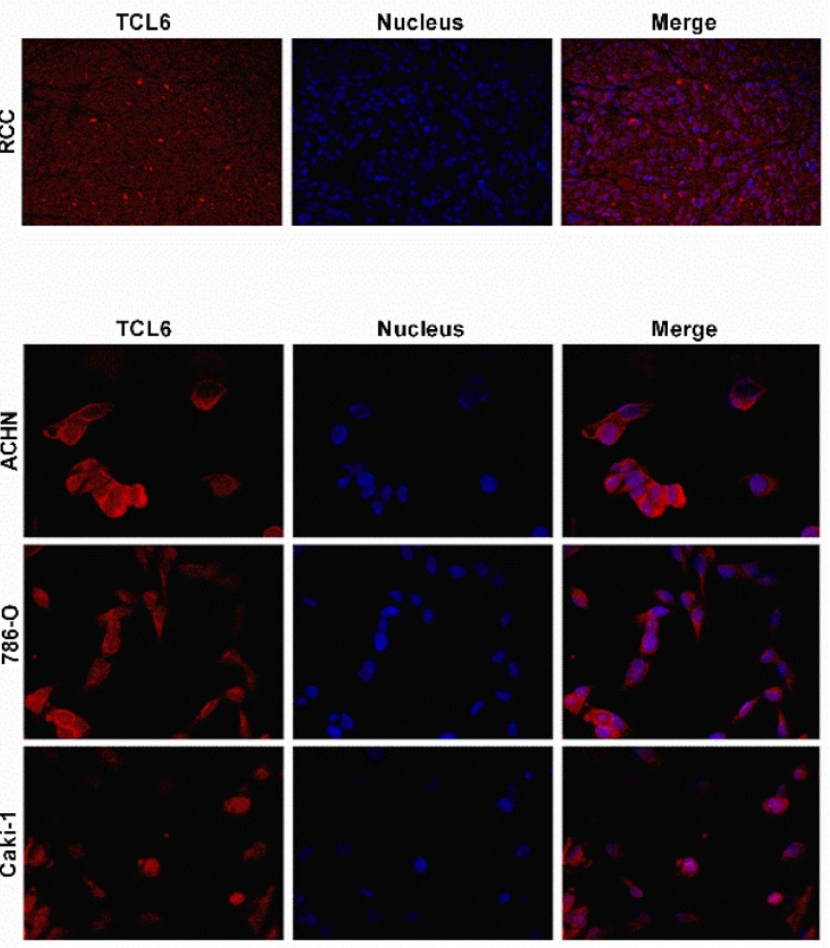

cytotoxicity effects with PTX in RCC cells, the apoptosis of 786-O and Caki-1 cells was evaluated by flow cytometer. Down-regulation of TCL6 expression in 786-O cells caused an inhibition of apoptosis compared with scrambled control 786-O (Fig.4A). cells, the apoptotic cell death apoptosis was significantly induced by overexpression of TCL6 (Fig.4B).
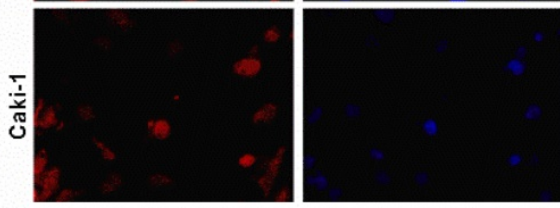

E

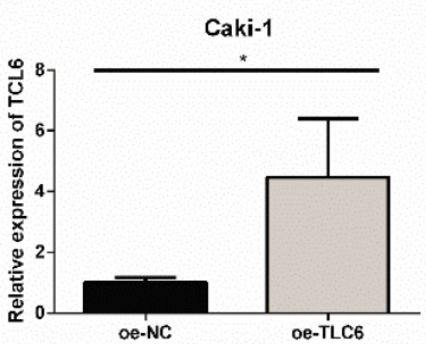

Figure 2. The relative expression levels of TCL6 in RCC tissues cells. A. Confocal FISH images exhibited expression and localization of TCL6 in RCC and adjacent normal renal tissues. TCL6 (red signal) was mainly located in the cytoplasm. Blue DAPI for nucleus staining. B. The levels of TCL6 expression was detected by FISH in HK-2 cells and 5 RCC cell lines. There were less red signals in 5 RCC cell lines compared to HK-2 cells. C. The basal expression of TCL6 in HK-2, 786-O, and Caki-1 cells was evaluated by qRT-PCR. D. 786-O cells were transfected with TCL6 siRNA1, siRNA2, siRNA3, and NC siRNA, respectively. qRT-PCR revealed the expression levels of TCL6 were significantly reduced by treatment with TCL6 siRNA 3. E. The expression levels of TCL6 were elevated in Caki-1 cells transfected with TCL6 overexpression plasmids compared to those cells were treated with the negative control plasmids. Data are shown as means \pm SD ( $=3$ ). FISH: fluorescence in situ hybridization; $* P<0.05$. 
A

786-0

0 nM

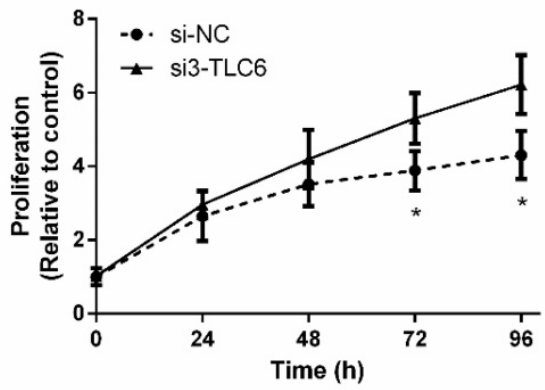

$50 \mathrm{nM}$

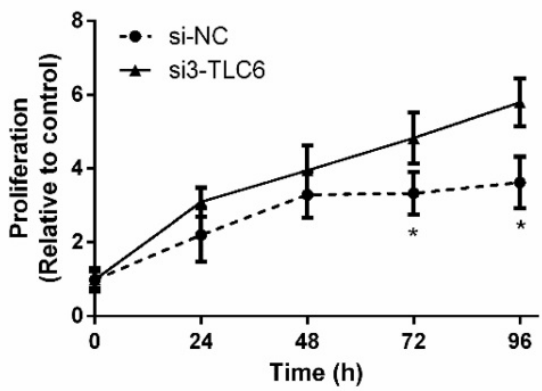

$100 \mathrm{nM}$

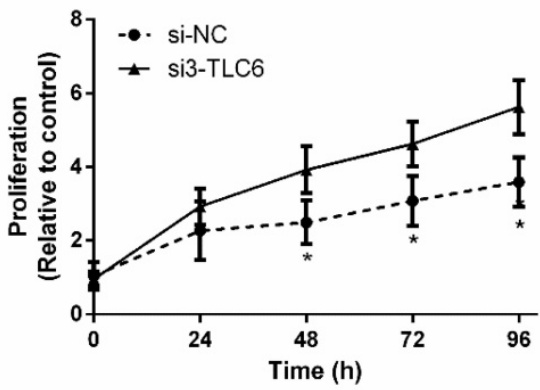

$200 \mathrm{nM}$

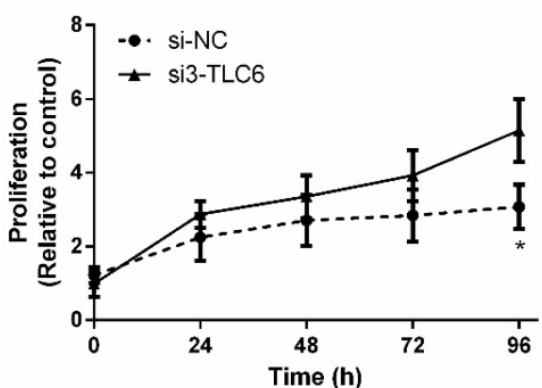

B

Caki-1

$0 \mathrm{nM}$

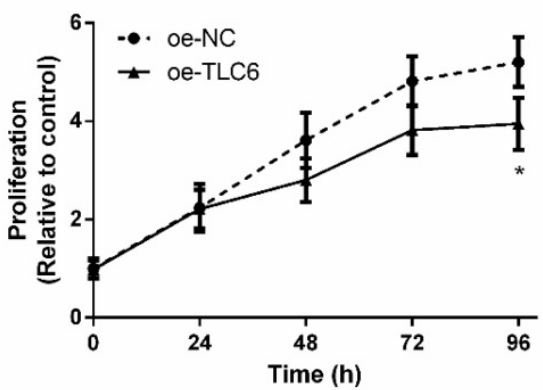

$50 \mathrm{nM}$

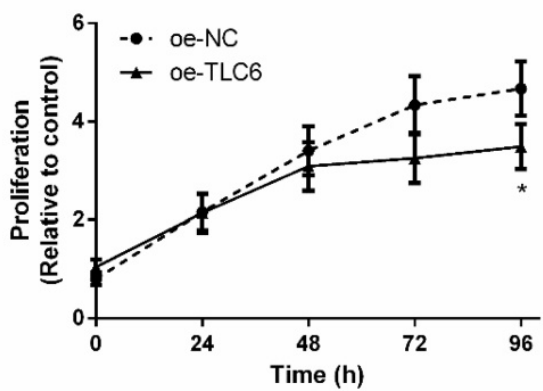

$100 \mathrm{nM}$

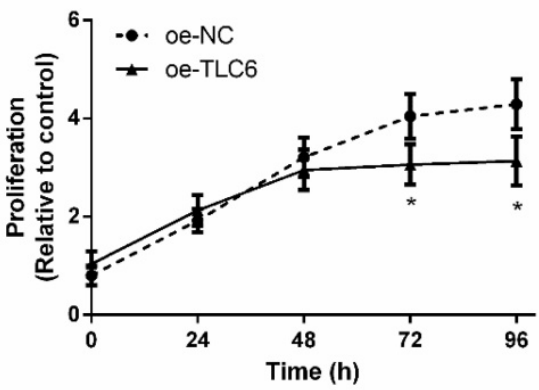

$200 \mathrm{nM}$

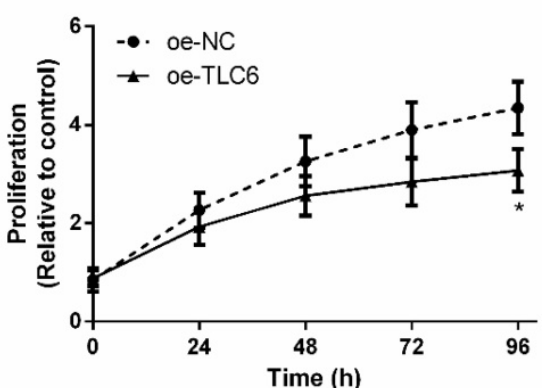

Figure 3. TCL6 expression is correlated with sensitization of RCC cells to PTX. The indicated concentrations of PTX were treated to two kinds of renal cancer cells for $0,24,48,72$, and $96 \mathrm{~h}$. A. The proliferation of TCL6-inhibited 786-O cells were significantly increased as compared to those 786-O cells treated with scramble control siRNAs (si-NC) with different levels of PTX concentration $(0,50,100,200 \mathrm{nM})$ at $96 \mathrm{~h}$. B. After incubation for $96 \mathrm{~h}$, the proliferation of TCL6-increased Caki-1 cells was significantly lower than those cells treated with empty pcDNA3.1 plasmid (oe-NC) with different concentrations of PTX. si-NC: 786-O cells treated with scrambled control siRNAs; si3-TCL6: 786-O cells treated with si3-TCL6 siRNAs; oe-NC: Caki-1 cells treated with empty pcDNA3.1 plasmid. oe-TCL6: Caki-1 cells treated with TCL6 overexpressing plasmids. Data are shown as means $\pm \mathrm{SD}$. $* P<0.05$.

\section{TCL6 sensitizes RCC cells to PTX through sponging miR-221}

The analysis of TANRIC platform showed that the level of miR-221 was significantly negatively correlated with TCL6 in renal cancer (Fig.5A). As shown in Fig.5B, miR-221 was elevated in RCC cells compared to HK2 cells. Silence of TCL6 increased the 
expression level of miR-221 in 786-O cells (Fig.5C), while overexpression of TCL6 decreased the expression level of miR-221 in Caki-1 cells (Fig.5D). According to miRcode, a map of putative microRNA target sites in the lncRNA, suggesting TCL6 is highly predicted to be a target of miR-221, and two potential binding sites were listed in Fig.5E. To investigate this hypothesis, the sequence of TCL6 was inserted into a

A
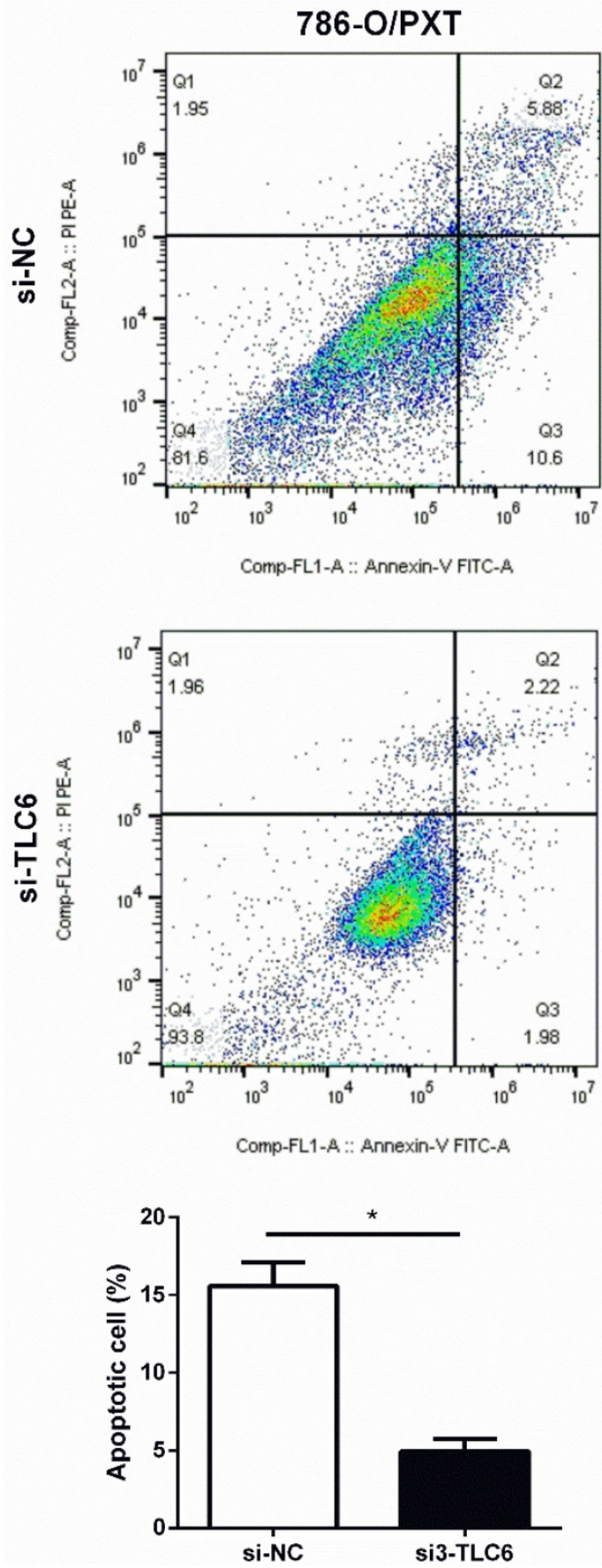

pmirGLO-Luc vector. As shown in Fig.5F, the luciferase activity was suppressed when HEK293 cells were co-transfected with the pmirGLO-TCL6-Luc vector and miR-221 mimics, compared with those cells co-transfected with the pmirGLO-TCL6-Luc vector and negative control mimics. These results suggest that miR-221 directly binds to TCL6.

B
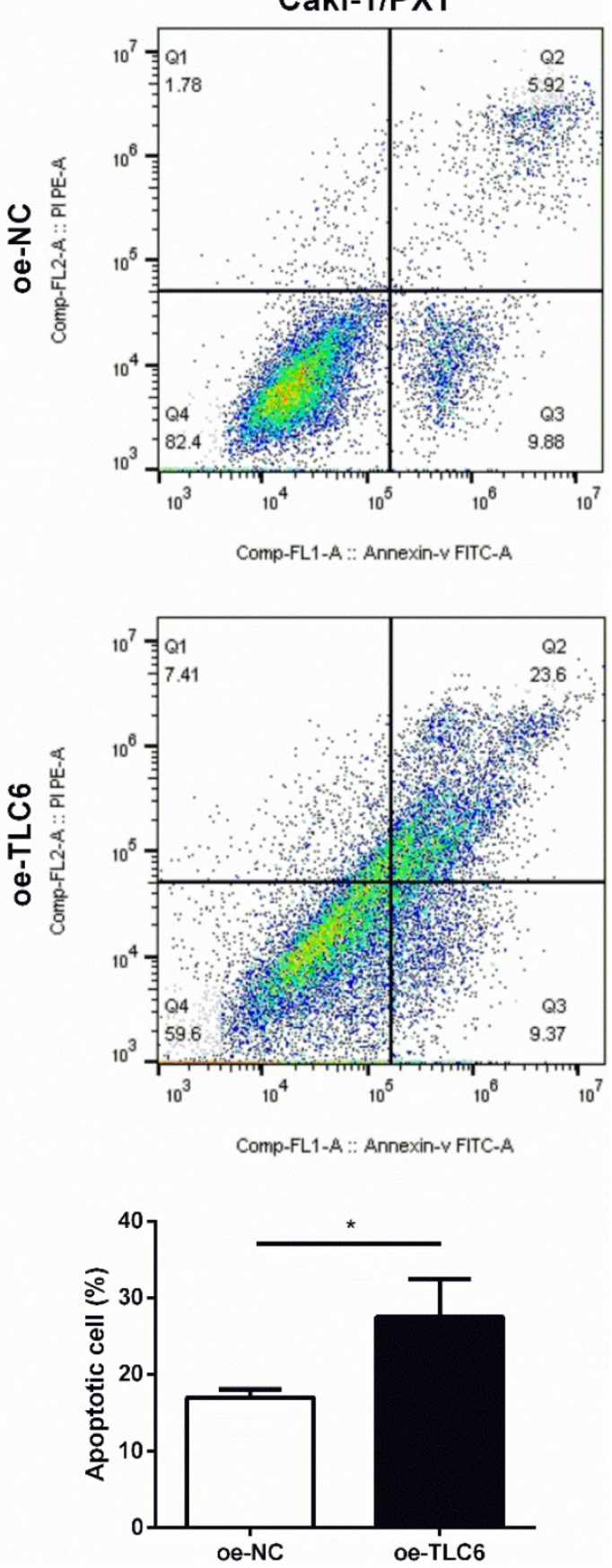

Figure 4. Modulation of PTX cytotoxicity by overexpression (oe-TCL-6) or inhibition (si3-TLC6) of TLC6. A. The apoptosis assays of 786-O cells were performed with (si3-TCL6) or without si3-TCL6 siRNAs (si-NC) under PTX treatments. B. Inversely, up-regulation of TCL6 decreased the apoptosis of Caki-1 cells compared to control cells (oe-NC). Data are shown as means $\pm S D(n=3)$. $* P<0.05$ 
A

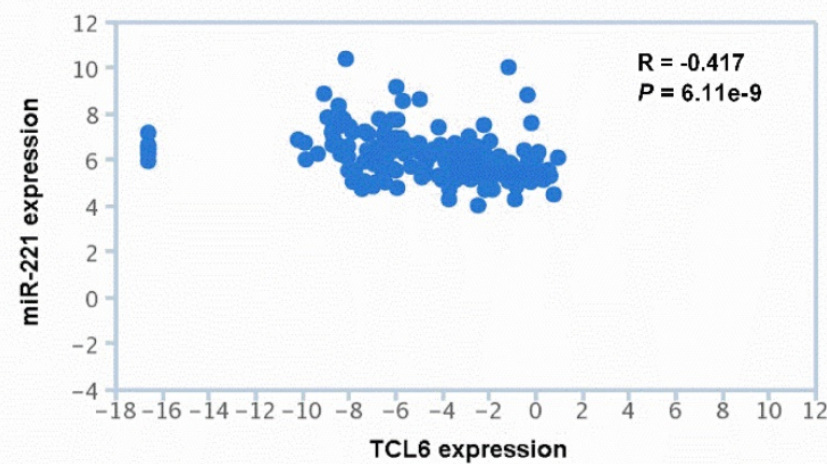

C

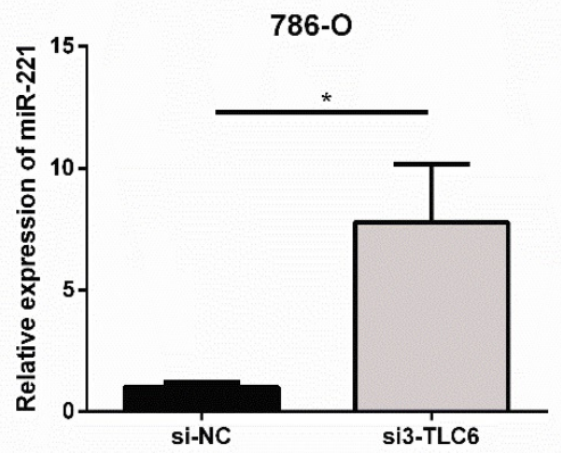

B

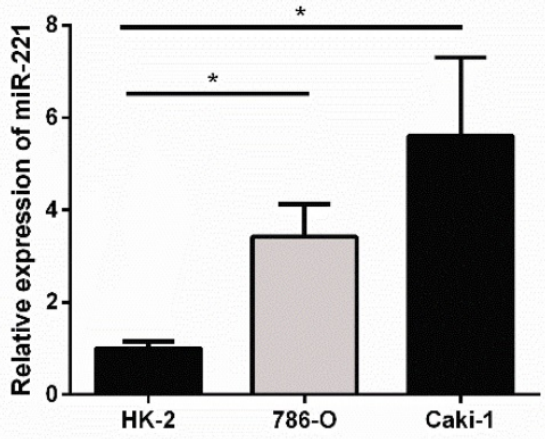

D

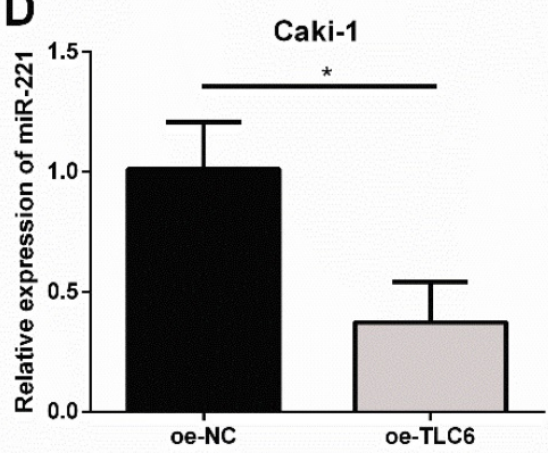

E

\begin{tabular}{lccccc}
\hline & Seed position & Seed type & Transcript region & Repeat & Conservation \\
\hline miR-221 & chr14:96130689 & 7-mer-m8 & ncRNA & no & $33 \%$ \\
miR-221 & chr14:96131118 & $8-\mathrm{mer}$ & ncRNA & yes & $33 \%$ \\
\hline
\end{tabular}

$\mathbf{F}$

HEK293

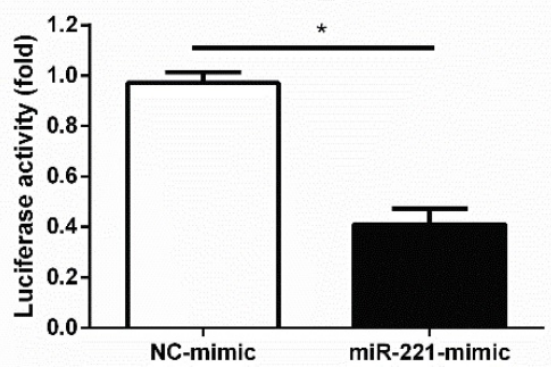

Figure 5. TCL6 sensitizes RCC cells to PTX through sponging miR-221. A. The level of miR-221 was negatively correlated with TCL6 expression in renal cancer, which was conducted via TANRIC platform. B. The basal expression of miR-221 in HK-2, 786-O, and Caki-1 cells were evaluated by qRT-PCR. C. The expression levels of miR-221 were detected by qRT-PCR after knockdown of TCL6 in 786-O cells. D. The expression level of miR-221 was further examined with or without upregulation of TCL6 using qRT-PCR. E. TCL6 contains two potential binding sites of miR-221. F. Relative luciferase activity in wild type of TCL6. The decrease in luciferase activity in the wild type indicated specific binding of miR-221 to its target site. Data are shown as means \pm SD. $* P<0.05$.

\section{Discussion}

RCC is known to be a heterogeneous group of cancers with disparate genetic and molecular alterations underlying the various histological subtypes with no effective therapeutic options. In this study, we found the expression deficiency of TCL6 (located on chromosome 14) was observed in RCC tissues through analysis of TCGA and GEO datasets, which was evidenced by multi-platform. Low level of TCL6 was associated with worse overall and disease-free survival of RCC patients. These findings were consistent with a previous study, TCL6 was reported to be negatively correlated with the T, N, M, and TNM stage and serve as an independent predictor via analysis of 71 pairs of RCC samples [19]. Here, we demonstrated that TCL6 mainly distributed in the cytoplasm. 
Further research is warranted into developing novel therapies in combination with standard chemotherapy regimens with greater efficacy than a single treatment. To further investigate whether TCL6 exerts synergistic cytotoxicity effects with PTX in RCC cells, we modified the expression level of TCL6 and analyzed its impacts on the cell viability and apoptosis of 786-O and Caki-1 cells. We demonstrate that inhibition of TCL6 reduces the cytotoxicity of PTX in RCC cells. Conversely, up-regulation of TCL6 is effective in sensitizing RCC response to PTX chemotherapy. Several functions have been identified for lncRNAs including the recruitment of chromatin-remodeling complexes to specific genomic loci that modify histone and RNA polymerase accessibility, miRNAs-sponge binding, and multiple epigenetic changes [20]. microRNA sponge is the main function of lncRNA in tumor chemotherapy. Such as, HOXA11-AS promotes cisplatin resistance of human LUAD cells via sponging miR-454-3p [21]. Linc00518 was demonstrated to decrease chemosensitivity to breast cancer cells through regulating the miR-199a [22] and UCA1 confers PTX resistance to ovarian cancer through silencing miR-129 [23]. Since LNCRNAs mainly act as modulators of other classes of RNAs, we thus focused on their sponging functions. Here, we found that down-regulation of TCL6 was accompanied by increasing the expression of miR-221, while overexpression of TCL6 inhibited the endogenous miR-221. A negative correlation between TCL6 and miR-221 in RCC tissues was confirmed by analysis of RNA-seq data derived from the TANRIC database. The previous study showed that elevated expression of miR-221 in tissues [24] and plasma [25] was associated with a poor prognosis in RCC patients. Moreover, inhibition of miR-221 resulted in significantly enhancing the therapeutic efficacy of PTX [26]. Thus, a possible mechanism explaining the association between higher TCL6 expression and higher PTX-induced cell apoptotic rate, was the sponging effect exerted by this LNCRNA on miR-221, which was confirmed by the luciferase reporter assay. Besides, we will further analyze the function of TCL6 by using RCC cell lines in the same design in the future study.

Collectively, our study elaborated a novel TCL6/miR-221 regulatory axis underlying PTX sensitivity of RCC cells, providing a potential therapeutic target for RCC.

\section{Abbreviations}

lncRNAs: long non-coding RNAs; PTX: paclitaxel; RCC: renal cancer cells; TCGA: The Cancer Genome Atlas; GEO: Gene Expression Omnibus;
FISH: Fluorescence in situ hybridization; TCL6: T-cell leukemia lymphoma 6; RCC: Renal cell carcinoma; GEPIA: Gene Expression Profiling Interactive Analysis; TANRIC: The Atlas of Noncoding RNAs in Cancer; ATCC: American Type Culture Collection; PI: propidium iodide.

\section{Competing Interests}

The authors have declared that no competing interest exists.

\section{References}

1. Ljungberg B, Campbell SC, Cho HY, Jacqmin D, Lee JE, Weikert S, et al. The epidemiology of renal cell carcinoma. Eur Urol. 2011; 60: 615-21.

2. Bechara GR, Júnior JADR, Gouveia HA, de Souza TAJOJoU. Metastasis to paranasal sinuses as the first presenting sign of renal cell carcinoma. Open Journal of Urology. 2012; 2: 28.

3. Patard J-J, Leray E, Rioux-Leclercq N, Cindolo L, Ficarra V, Zisman A, et al. Prognostic value of histologic subtypes in renal cell carcinoma: a multicenter experience. J Clin Oncol. 2004; 23: 2763-71.

4. Kanesvaran R, Tan M-HJJoc. Targeted therapy for renal cell carcinoma: the next lap. J Carcinog. 2014; 13

5. Derosa L, Albiges L, Massard C, Loriot Y, Fizazi K, Escudier BJEoods. Safety of available treatment options for renal cell carcinoma. Expert Opin Drug Saf. 2016; 15: 1097-106.

6. Beksac AT, Paulucci DJ, Blum KA, Yadav SS, Sfakianos JP, Badani KK. Heterogeneity in renal cell carcinoma. Urol Oncol. 2017. p. 507-15.

7. Hong B, Yang Y, Guo S, Duoerkun S, Deng X, Chen D, et al. Intra-tumour molecular heterogeneity of clear cell renal cell carcinoma reveals the diversity of the response to targeted therapies using patient-derived xenograft models. Oncotarget. 2017; 8: 49839.

8. Massari F, Ciccarese C, Bria E Porta C La Russa F, Knuutila S, et al. Reprofiling metastatic samples for chromosome $9 p$ and $14 q$ aberrations as a strategy to overcome tumor heterogeneity in clear-cell renal cell carcinoma. Appl Immunohistochem Mol Morphol. 2017; 25: 39-43.

9. Grimaldi G, Rajendra S, Matthews JJB, communications br. The aryl hydrocarbon receptor regulates the expression of TIPARP and its cis long non-coding RNA, TIPARP-AS1. Biochem Biophys Res Commun. 2018; 495: 2356-62.

10. Dasgupta P, Kulkarni P, Majid S, Varahram S, Hashimoto Y, Bhat NS, et al. MicroRNA-203 inhibits long noncoding RNA HOTAIR and regulates tumorigenesis through epithelial-to-mesenchymal transition pathway in renal cell carcinoma. Mol Cancer Ther. 2018: molcanther. 0925.2017.

11. Hirata H, Hinoda Y, Shahryari V, Deng G, Nakajima K, Tabatabai ZL, et al. Long noncoding RNA MALAT1 promotes aggressive renal cell carcinoma through Ezh2 and interacts with miR-205. Cancer Res. 2015.

12. Zhou S, Wang J, Zhang ZJJocr, oncology c. An emerging understanding of long noncoding RNAs in kidney cancer. J Cancer Res Clin Oncol. 2014; 140: 1989-95.

13. Chen D, Zhu M, Su H, Chen J, Xu X, Cao CJJocp. LINC00961 restrains cancer progression via modulating epithelial-mesenchymal transition in renal cell carcinoma. J Cell Physiol. 2018.

14. Scripture CD, Figg WD, Sparreboom AJT, management cr. Paclitaxel chemotherapy: from empiricism to a mechanism-based formulation strategy. Ther Clin Risk Manag. 2005; 1: 107.

15. Tomczak K, Czerwińska P, Wiznerowicz MJCo. The Cancer Genome Atlas (TCGA): an immeasurable source of knowledge. Contemp Oncol (Pozn). 2015; 19: A68.

16. Tang Z, Li C, Kang B, Gao G, Li C, Zhang ZJNar. GEPIA: a web server for cancer and normal gene expression profiling and interactive analyses. Nucleic Acids Res. 2017; 45: W98-W102.

17. Li J, Han L, Roebuck P, Diao L, Liu L, Yuan Y, et al. TANRIC: an interactive open platform to explore the function of IncRNAs in cancer. Cancer Res. 2015; 0273.2015

18. Ding F, Tang H, Nie D, Xia LJOl. Long non-coding RNA Fer-1-like family member 4 is overexpressed in human glioblastoma and regulates the tumorigenicity of glioma cells. Oncol Lett. 2017; 14: 2379-84.

19. Su H, Sun T, Wang H, Shi G, Zhang H, Sun F, et al. Decreased TCL6 expression is associated with poor prognosis in patients with clear cell renal cell carcinoma. Oncotarget. 2017; 8: 5789.

20. Iyer MK, Niknafs YS, Malik R, Singhal U, Sahu A, Hosono Y, et al. The landscape of long noncoding RNAs in the human transcriptome. Nat Genet. 2015; 47: 199.

21. Zhao X, Li X, Zhou L, Ni J, Yan W, Ma R, et al. LncRNA HOXA11-AS drives cisplatin resistance of human LUAD cells via modulating miR-454-3p/Stat3. Cancer Sci. 2018; 109: 3068. 
22. Chang L, Hu Z, Zhou Z, Zhang HJCP, Biochemistry. Linc00518 contributes to multidrug resistance through regulating the MiR-199a/MRP1 axis in breast cancer. Cell Physiol Biochem. 2018; 48: 16-28.

23. Wang J, Ye C, Liu J, Hu YJB, communications br. UCA1 confers paclitaxel resistance to ovarian cancer through miR-129/ABCB1 axis. Biochem Biophys Res Commun. 2018; 501: 1034-40.

24. Gu L, Li H, Chen L, Ma X, Gao Y, Li X, et al. MicroRNAs as prognostic molecular signatures in renal cell carcinoma: a systematic review and meta-analysis. Oncotarget. 2015; 6: 32545.

25. Teixeira AL, Ferreira M, Silva J, Gomes M, Dias F, Santos JI, et al. Higher circulating expression levels of miR-221 associated with poor overall survival in renal cell carcinoma patients. Tumour Biol. 2014; 35: 4057-66.

26. Zhou Z, Kennell C, Lee J-Y, Leung Y-K, Tarapore PJNN, Biology, Medicine. Calcium phosphate-polymer hybrid nanoparticles for enhanced triple negative breast cancer treatment via co-delivery of paclitaxel and miR-221/222 inhibitors. Nanomedicine. 2017; 13: 403-10. 